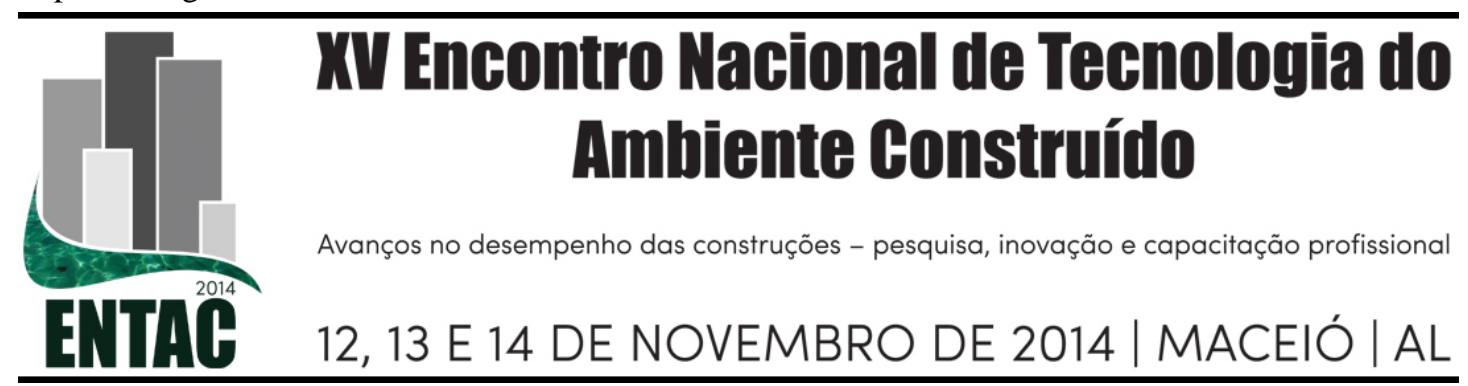

\title{
BIM NO SUPORTE A AVALIAÇÃO PÓS-OCUPAÇÃO
}

SALES, Adriano de Alencar (1); RUSCHEL, Regina C.(2)

(1) PPGEC-UNICAMP, e-mail: adriarchi@hotmail.com

(2) FEC-UNICAMP, e-mail: ruschel@fec.unicamp.br

\begin{abstract}
RESUMO
Esta pesquisa avalia como tornar mais eficiente a reutilização dos dados coletados em APO para viabilizar o processo de retro-alimentação fazendo uso da Tecnologia da Informação na Construção (TIC). Sugere-se que o modelo de informação da edificação incorpore os dados coletados em APO e que por meio da interoperabilidade este seja compartilhado entre projetistas e assim utilizado em novos empreendimentos. Mas de que forma? E o quanto o modelo de informação é capaz de absorver o tipo de informação coletado nestes estudos? Uma vez inseridos no modelo de informação como podem ser utilizados? A pesquisa foi exploratória e classificada como um estudo de campo. O estudo de campo desenvolveu-se a partir das seguintes etapas: domínio do problema, formulação, desenvolvimento e pré- testes, aplicação e análise. O domínio do problema abrangeu os três temas focais estudados, sendo estes: Avaliação Pós- Ocupação, Modelo Paramétrico BIM (Building Information Modeling) e processos de projeto. O estudo foi validado num conjunto habitacional específico. Foram analisados APOs anteriormente realizadas para subsidiar exemplos concretos de dados coletados. O modelo de informação do conjunto habitacional foi testado em plataformas distintas, sendo avaliada como base de interoperabilidade o formato aberto IFC. Observou-se que o componente da categoria zona no modelo de informação é o mais apropriado para a introdução e manuseio de propriedades como: identificação e satisfação. Foi possível replicar a tabulação dos dados coletados na APO pela extração dos dados inserido no modelo BIM.
\end{abstract}

Palavras-chave: BIM, Avaliação Pós-Ocupação, Tecnologia da Informação na Construção.

\begin{abstract}
This research examines how to optimize the reuse of data collected in a Post-Occupation Evaluation (POE) to enable the design feedback making use of Information Technology in Construction. It is suggested to incorporate to the Building Information Model the data collected in such evaluations and that through interoperability shared information between designers and in new developments. But how? How should the information model absorb the type of information collected in these studies? Once entered into the model as information how can it be used? The research was exploratory and classified as a field study. The field study was developed from the following steps: understanding the problem domain, formulation of a solution, development and pre-testing, implementation and analysis. The problem domain involved three focal themes: Post-Occupancy Evaluation, Building Information Modeling and design processes. The study was validated in for a specific housing development. POEs previously undertaken where considered subsidizing specific examples of data collected. The information model of the housing development was tested on different platforms considering best performance in terms of interoperability by IFC format. It was observed that the component category Zone was the most suitable for the introduction and handling data such as identification and satisfaction. It was possible to replicate the tabulation of data collected in the POE with the extraction of data inserted into the BIM model.
\end{abstract}

Keywords: BIM, Post-Occupation Evaluation, Information technology in construction. 


\section{INTRODUÇÃO}

A arquitetura pública relativa a hospitais, escolas e moradias, costuma ser encomendada por clientes que não serão os usuários diretos. Desta forma, a comunicação entre projetista e usuário final é indireta. Nesse contexto, a Avaliação Pós-Ocupação (APO) aparece como eficaz ferramenta de avaliação sistemática do ambiente construído, emulando uma comunicação entre projetista e usuário. APO é conceituada como sendo uma "metodologia" que pretende diagnosticar os aspectos positivos e negativos do ambiente em uso a partir da avaliação de fatores técnicos, funcionais, econômicos, estéticos e comportamentais, tendo em vista tanto a opinião dos técnicos, projetistas e clientes, como também dos usuários (ORNSTEIN; ROMÉRIO, 1992).

Para Preiser, Rabinowitz e White (1988) existem três níveis de APO: indicativa, investigativa e de diagnóstico; implicando em três temporalidades de curto, médio e longo prazos das implicações de aplicação das recomendações resultantes no ambiente avaliado. O diagnóstico resultante da APO também é insumo na fase de planejamento estratégico para novos empreendimentos similares ao avaliado devido ao ciclo de retroalimentação no processo de projeto (SILVA; SOUZA, 2003).

A questão abordada neste artigo é como tornar mais eficiente a reutilização dos dados coletados para viabilizar a retroalimentação em processo de projeto utilizando BIM. Pretende-se contribuir para o desenvolvimento de soluções inovadoras em tecnologia da informação e comunicação aplicadas à construção no contexto da operação e planejamento de edificações. Desta forma, o objetivo do estudo apresentado é propor diretrizes para integração de Building Information Modeling (BIM) e Avaliação PósOcupação (APO), tornando mais eficiente a coleta dos dados de APO, registro e reutilização em processo de projeto de Habitação de Interesse Social (HIS).

\section{AVALIAÇÃO PÓS- OCUPAÇÃO}

Estudos de Avaliação Pós-Ocupação deveriam informar como um edifício está se desempenhando na prática (LEAMAN; STEVENSON; BORDASS, 2010). Este desempenho pode ser avaliado por três perspectivas: do ocupante, do ambiente e econômica. O objetivo final de uma APO é o aprendizado. Este aprendizado pode beneficiar um projeto específico ou contribuir para a construção de um conhecimento mais geral (PREISER; RABINOWITZ;WHITE, 1988). Segundo Zimring (2002, p. 310) a APO se diversificou com o tempo e inclui uma prática variada, envolvendo "debates sobre o desenvolvimento de conhecimentos e métodos das ciências sociais e humanas".

Não existe um modelo de APO que se destaca. Entretanto, o modelo proposto por Preiser, Rabinowitz e White (1988) foi selecionado para este estudo pela sua característica sistêmica. Os autores propõem um modelo de processo para APO que inclui três fases: de planejamento, condução e aplicação (Figura 1). No planejamento realiza-se reconhecimento do caso de estudo, verifica-se a viabilidade do estudo, definem-se recursos e instrumentos da pesquisa. $\mathrm{Na}$ fase de condução, executa-se o pré-teste dos recursos e instrumentos da pesquisa, a coleta efetiva dos dados e a análise dos mesmos. $\mathrm{Na}$ fase de aplicação reportam-se os resultados, recomendam-se ações e monitoram-se os impactos da aplicação destas ações. 
Figura 1 - Etapas tradicionais da Avaliação Pós-Ocupação

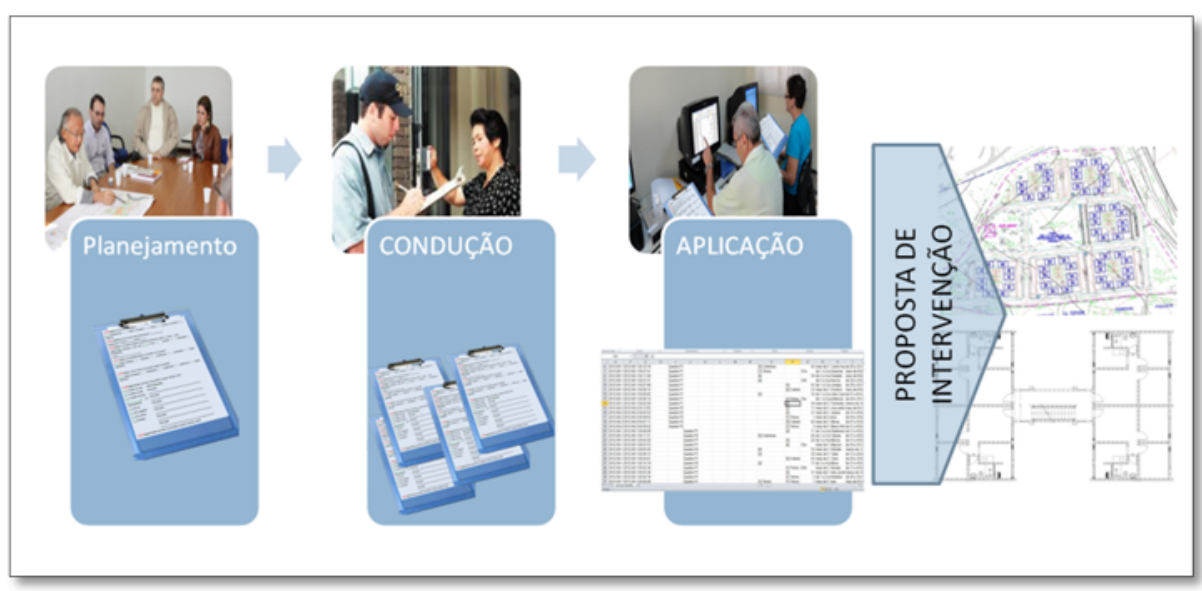

Fonte: $\mathrm{O}$ autor

\section{MODELAGEM DA INFORMAÇÃO DA CONSTRUÇÃO (BIM)}

BIM é visto como um sistema para representar e comunicar informação gerada durante o ciclo de vida de uma edificação consistindo em uma tecnologia emergente e uma mudança processual para os setores de Arquitetura, Engenharia, Construção e Operações - AECO SUCCAR (2009). Outros autores EASTMAN et al. (2008) também ressaltam que BIM é uma tecnologia de modelagem e requer processos associados usados na produção, comunicação e análise de modelos de edifícios. Trata-se de um paradigma que apresenta duas características principais que o diferenciam da tecnologia CAD comumente em uso por profissionais de AEC. Estas características são a modelagem orientada a objetos e a interoperabilidade. Por meio da modelagem paramétrica os objetos são representados por parâmetros e regras associados à geometria que, por sua vez, são integradas com o propósito de evitar inconsistências, independentemente de como são apresentadas (2D ou 3D). São consideradas ferramentas de autoria BIM as ferramentas que permitem aos usuários elaborarem modelos que sejam objetos paramétricos.

Outra característica do paradigma BIM é a interoperabilidade que consiste na propriedade de intercambiar modelos de informação por meio da importação (para criação e edição de projeto) e exportação de dados entre aplicativos, o que garante uma prática integrada entre um time de trabalho. Para que isso ocorra, é necessária a implementação de um protocolo de troca de dados nos aplicativos e nos processos do projeto, onde se enquadram os formatos de troca de dados de domínio público, entre outros existentes, cujos principais para construção civil são: Industry Foundation Classes (IFC) e CIMSteel Integration Standards, release 2 (CIS/2) (EASTMAN et al. (2008).

\section{MÉTODO DE PESQUISA}

A pesquisa proposta foi exploratória e classificada como um estudo de campo, já que os procedimentos adotados visam o aprofundamento das questões propostas. Integraram-se os resultados de APO nos modelos de informações associados e criaram-se mecanismos para sua visualização, consulta, validação de regras e uso no processo de projeto. O estudo de campo desenvolveu-se seguindo as etapas de pesquisa: domínio do problema, formulação, desenvolvimento e pré-testes, aplicação e análise (GIL, 2002, p. 129-135).

A etapa de domínio do problema desenvolveu-se por meio da revisão bibliográfica e fundamentação teórica. Avaliou-se: (i) como se desenvolvem APOs e como são 
apresentados os seus resultados; (ii) qual é a estruturação e composição de um modelo de informação em BIM e (iii) quais as possibilidades de integração, manuseio, troca e reutilização dos dados de APO em ambiente colaborativo tendo como base o modelo paramétrico, e quais as questões em processo de projeto que são respondidas por este procedimento. Uma síntese desta etapa foi apresentada na seção 2 deste artigo.

$\mathrm{Na}$ etapa de formulação avaliou-se a estrutura do modelo da edificação quanto ao seu comportamento diante das possibilidades de introdução e manuseio de propriedades, quais os componentes do modelo são mais apropriados para estas possíveis ações e qual a melhor escala a ser trabalhada (apenas um apartamento tipo, um pavimento, um bloco inteiro ou uma quadra). A partir de uma APO realizada nos moldes de Preiser, Rabinowitz e White (1988) avaliou-se conjuntamente, a estrutura do formulário considerando a composição de suas questões (perguntas e respostas), qual o foco de cada questão e objetivo (se é a satisfação e percepção do usuário, se é a detecção de patologias, etc.) para que a partir desta decomposição da estrutura do formulário fosse possível identificar no modelo BIM da edificação uma correspondência para integração de questões e componentes.

$\mathrm{Na}$ etapa de desenvolvimento identificou-se e implementou-se na ferramenta de autoria BIM Archicad as propriedades a serem acrescidas ao modelo de informação da edificação para incorporar a dados coletados na APO (numeração, perguntas e respostas).

Na etapa de pré-teste foi utilizado o software Solibri Model Checker para validar e avaliar a integridade do modelo em formato de arquivo IFC. Nesta etapa, foram realizadas exportações em arquivos IFC para verificar a integridade e manuseio do modelo de informação, preservando as propriedades inseridas na etapa anterior no modelo da edificação (mapeamento e introdução das questões no modelo paramétrico).

Na fase de análise buscou-se replicar a tabulação dos dados coletados na APO pela extração dos dados inserido no modelo BIM.

\section{DESENVOLVIMENTO}

\subsection{APO realizada de modo tradicional}

Realizou-se uma APO num conjunto habitacional específico para subsidiar a aplicação da proposta de integração de APO e BIM. Este exercício também foi considerado como uma ação para o domínio do problema. Seguindo o modelo de processo para APO proposto por Preiser, Rabinowitz e White (1988) desenvolveu-se uma avaliação indicativa (nível 1) fazendo uso dos métodos de coleta de dados com formulários e observação.

A fase de planejamento foi desenvolvida em conjunto os grupos de pesquisa GMIC e HABITARES $^{1}$. A partir do reconhecimento do local, por meio de estudos ali realizados anteriormente (KOWALTOWSKI et al., 2005; GRANJA et al., 2009) e considerando as demandas de avaliação de desempenhos ambientais, de acessibilidade, de durabilidade de materiais e componentes e reformas no conjunto habitacional em estudo pelos grupos de pesquisa GMIC e HABITARES desenvolveu-se os instrumentos de coleta de dados: formulários e roteiros para observação.

\footnotetext{
${ }^{1}$ GMIG - Grupo de Pesquisa em Modelagem da Informação e Colaboração Digital \& HABITARES Grupo de Pesquisa Habitar Cidades Vivas e + Sustentáveis. Ambos da Faculdade de Engenharia Civil, Arquitetura e Urbanismo da UNICAMP.
} 
Os formulários registram níveis de satisfação dos moradores quanto aos apartamentos por meio de questões, com alternativas pré-estabelecidas, referentes a conforto térmico, acústico, patologias, locomoção e acessibilidade, modificações realizadas e priorização de desejos quanto as questões avaliadas (quadro 1). Ao final da entrevista com o morador, tomando o formulário como roteiro, pedia-se permissão para visitação ao apartamento com o objetivo de registrar manifestações patológicas em cada um dos cômodos. Buscava-se levantar nos componentes de piso, parede, teto, porta, janela problemas do tipo: rachadura, descascamento, desgaste, umidade, infiltração, ferrugem e falta ou quebra de partes. Os entrevistados eram caracterizados por: nome, sexo e escolaridade. Registrava-se também quantas pessoas moravam no apartamento e se havia entre os moradores portador de deficiência.

\section{Quadro 1 - Questões de satisfação da APO realizada no conjunto habitacional}

\begin{tabular}{|l|l|}
\hline QUESTÃO & RESPOSTA - ALTERNATIVAS \\
\hline $\begin{array}{l}\text { 1.Qual a sua satisfação quanto ao } \\
\text { barulho no seu quarto? }\end{array}$ & $\begin{array}{l}\text { Muito satisfeito; Satisfeito; Indiferente; Insatisfeito; Muito } \\
\text { insatisfeito }\end{array}$ \\
\hline $\begin{array}{l}\text { 2. Como você se sente no seu } \\
\text { quarto quanto ao barulho?: }\end{array}$ & $\begin{array}{l}\text { Muito barulhento; Barulhento; Indiferente; Silencioso; Muito } \\
\text { silencioso }\end{array}$ \\
\hline $\begin{array}{l}\text { 3.Qual barulho que mais te } \\
\text { incomoda no quarto durante o } \\
\text { dia? }\end{array}$ & $\begin{array}{l}\text { Vizinho de cima; Vizinho do lado; Vizinho de baixo; Do } \\
\text { estacionamento; Da rua; Do campinho; Da rodovia; Nenhum }\end{array}$ \\
\hline $\begin{array}{l}\text { 4.Qual barulho que mais te } \\
\text { incomoda no quarto durante a } \\
\text { noite? }\end{array}$ & $\begin{array}{l}\text { Vizinho de cima; Vizinho do lado; Vizinho de baixo; Do } \\
\text { estacionamento; Da rua; Do campinho; Da rodovia; Nenhum }\end{array}$ \\
\hline $\begin{array}{l}\text { 5.Qual a sua satisfação quanto a } \\
\text { temperatura no seu quarto? }\end{array}$ & $\begin{array}{l}\text { Muito satisfeito; Satisfeito; Indiferente; Insatisfeito; Muito } \\
\text { insatisfeito }\end{array}$ \\
\hline $\begin{array}{l}\text { 6.Como você se sente quanto a } \\
\text { temperatura de seu quarto? }\end{array}$ & Muito Quente; Quente;Agradável;Frio;Muito frio \\
\hline $\begin{array}{l}\text { 7.Qual a sua satisfação para se } \\
\text { locomover até a cidade? }\end{array}$ & $\begin{array}{l}\text { Muito satisfeito; Satisfeito; Indiferente; Insatisfeito; Muito } \\
\text { insatisfeito }\end{array}$ \\
\hline $\begin{array}{l}\text { 8.Como você vai para o seu } \\
\text { trabalho? }\end{array}$ & A pé; Bicicleta; Ônibus; Carro; Moto; Fretado; Van; Outro: \\
\hline
\end{tabular}

Fonte: $\mathrm{O}$ autor

A tabulação dos dados coletados na fase de condução foi desenvolvida numa Iniciação Científica (FUGIMORI, 2012) gerando planilhas .XLS, Microsoft Excel (Figura 2) e análise dos mesmos (Figura 3). Na planilha da Figura 2, as questões são dispostas nas colunas identificadas por palavras-chave e uma linha representa um formulário respondido.

\subsection{Modelo Paramétrico}

Os modelos de informação paramétricos BIM se distinguem dos modelos CAD basicamente por não representarem objetos com geometria e propriedades fixas, mas por representarem objetos através de parâmetros e regras, que são expressões que relacionam os objetos, permitindo que sejam atualizados automaticamente de acordo com o controle ou mudança de contexto. 
Figura 2 - Planilha resultante da tabulação dos dados coletados na APO no conjunto habitacional CAMPINAS F

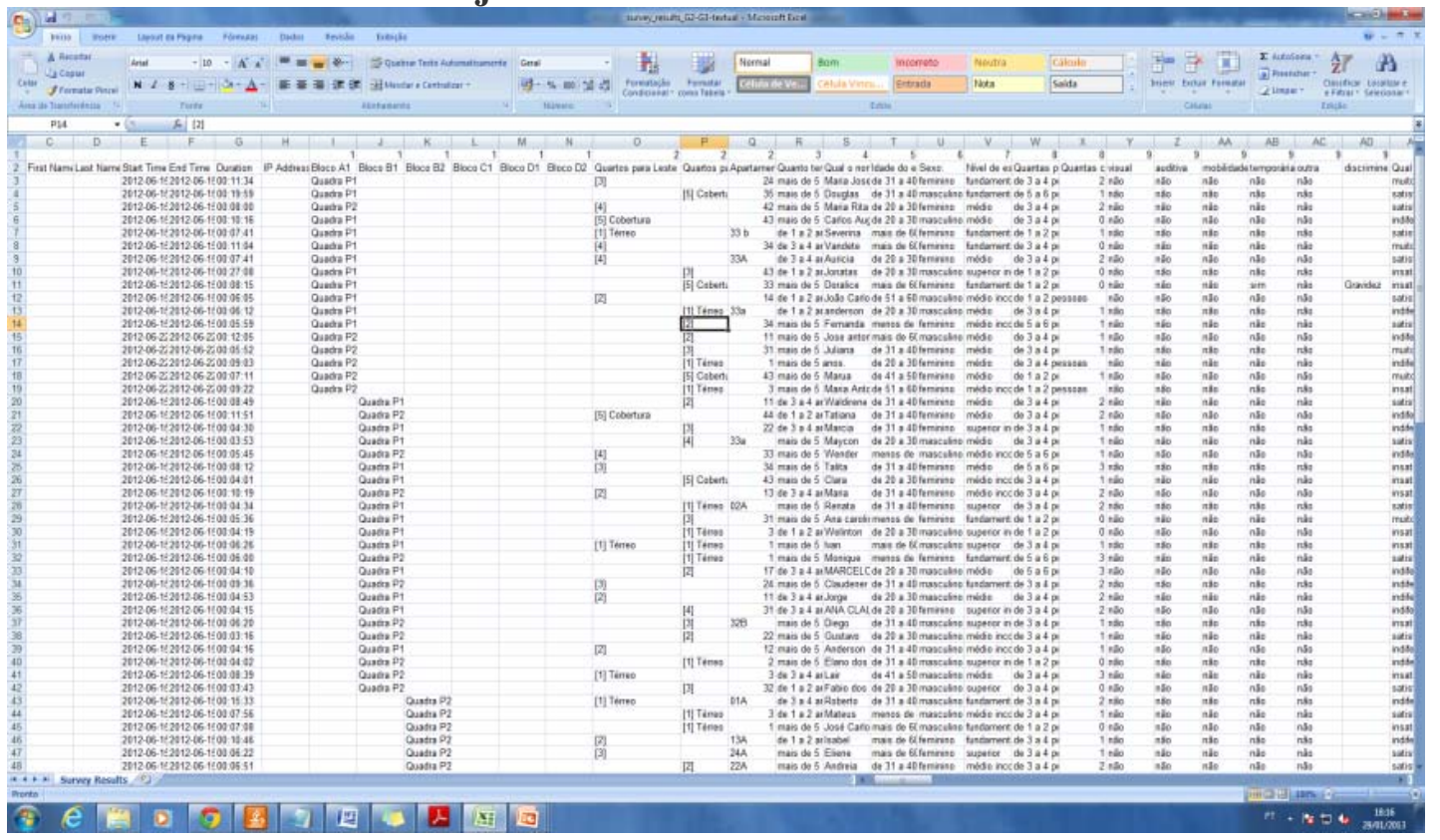

Fonte: Fujimore (2012)

Figura 3 - Exemplo da análise dos dados coletados quanto ao conforto acústico nos quartos: satisfação e fonte dos ruídos

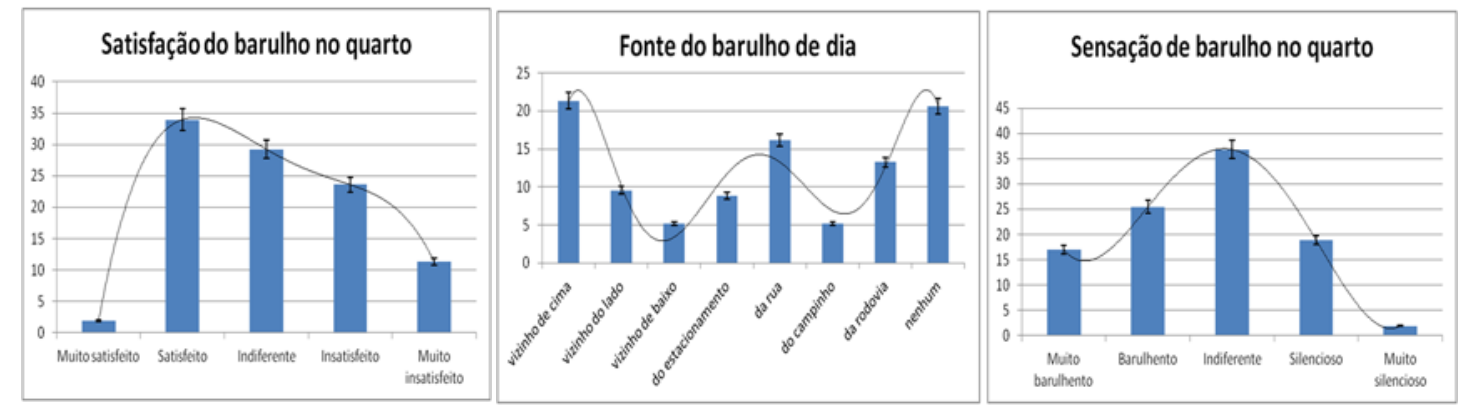

Fonte: Fujimore (2012)

Objetos paramétricos personalizados permitem a modelagem de geometrias complexas, que anteriormente não seriam possíveis ou simplesmente impraticáveis. Em outras indústrias, as empresas utilizam modelagem paramétrica para desenvolver suas próprias representações de objetos e refletir sobre conhecimento corporativo e melhores práticas (EASTMAN, 1999). O mesmo ocorre na indústria da arquitetura e construção, onde empresas de softwares BIM, desenvolveram um conjunto base, construindo classes de objetos para os usuários como portas, janelas, lajes, paredes, pilares, vigas, coberturas metálicas, etc (Figura 5). Atualmente, no Brasil, as maiores empresas de softwares que geram modelos BIM são a Autodesk e a Graphisoft, oferecendo respectivamente os softwares Revit e Archicad.

Para definir a plataforma utilizada nesta etapa do estudo, um modelo base simplificado (edificação simples), foi analisado nas duas plataformas mais utilizadas no mercado brasileiro, o Revit e o Archicad. Nesta avaliação comparativa foram levados em consideração alguns critérios, tais como o tamanho do arquivo nativo, o tamanho e 
consistência dos arquivos IFC gerados, e a comunicabilidade com plataforma Microsoft Excel (base de dados da compilação dos formulários da APO).

Figura 4 - Exemplos de famílias de objetos e modelo paramétrico (Campinas F)

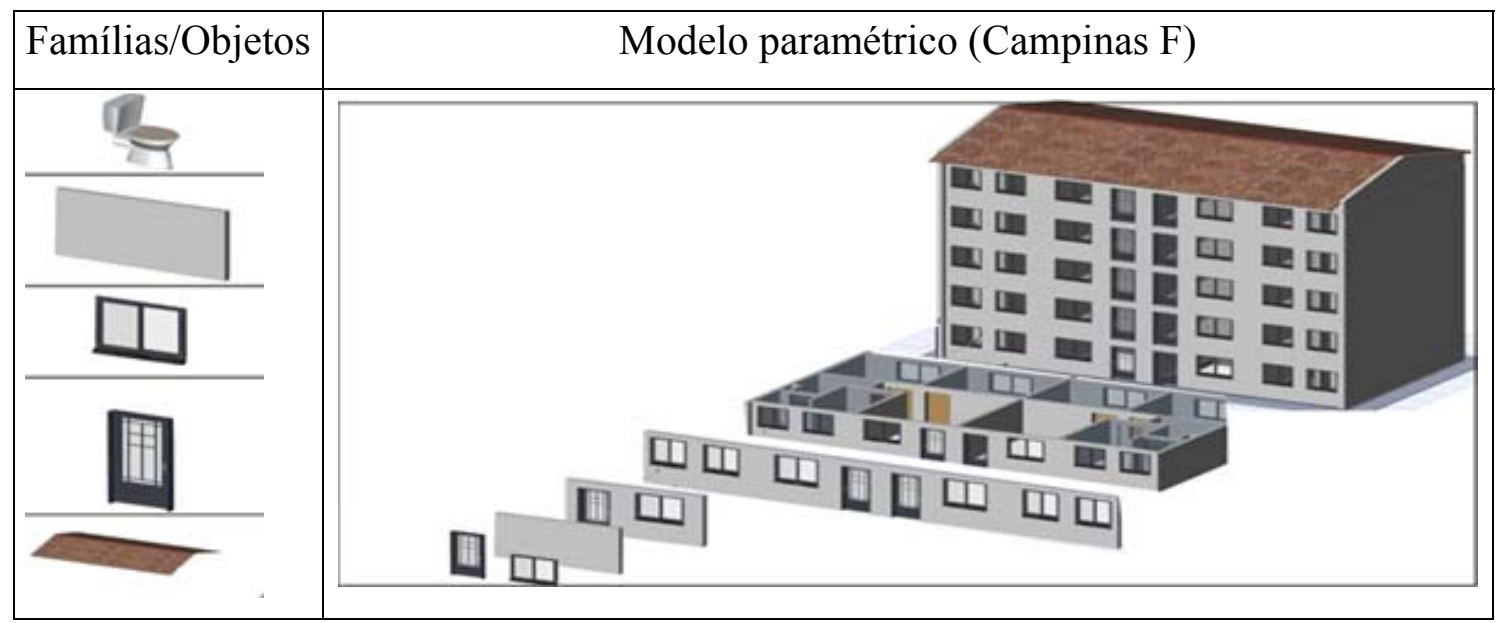

Fonte: $\mathrm{O}$ autor.

Dos resultados obtidos nesta análise comparativa, observou-se que o arquivo nativo gerado pelo Archicad (. PLN) é mais compacto. Levando-se em consideração o tamanho dos arquivos integrais do Conjunto Habitacional em estudo $(87.860 \mathrm{~Kb})$, o arquivo mais compacto, para esta fase é o ideal para manuseio, simulações, etc. Os arquivos. IFC gerados a partir dos modelos base (Archicad. PLN e Revit.RVT), se comportaram de maneira distintas ao serem avaliados pelo Software Solibri. E arquivos. ..XLS são fundamentais para maior facilidade de tocas de arquivos bases da APO (formulários. XLS) e o modelo paramétrico (Quadro 2).

Quadro 2 - Categorias das questões

\begin{tabular}{|c|c|c|c|c|c|}
\hline $\begin{array}{c}\text { Software } \\
\text { Arquivo } \\
\text { nativo }\end{array}$ & $\begin{array}{l}\text { Tamanho } \\
\text { arquivo } \\
\text { base } \\
\text { (Kb) }\end{array}$ & $\begin{array}{c}\text { Tamanho } \\
\text { arquivo } \\
\text { Conjunto } \\
\text { Habitacional } \\
\text { em estudo }\end{array}$ & \multicolumn{2}{|c|}{$\begin{array}{l}\text { Consistêrncia do Arquivo IFC } \\
\text { gerados } \\
\text { Visualizados no Solibri }\end{array}$} & $\begin{array}{c}\text { Exporta } \\
\text { Lista .XLS }\end{array}$ \\
\hline $\begin{array}{l}\text { Archicad } \\
. \text { PLN }\end{array}$ & $1.615 \mathrm{~Kb}$ & $87.860 \mathrm{~Kb}$ & $\begin{array}{c}\text { Sim } \\
\text { Conssistente }\end{array}$ & & sim \\
\hline $\begin{array}{l}\text { Revit } \\
\text {.RVT }\end{array}$ & $2.848 \mathrm{~Kb}$ & $154.938 \mathrm{~Kb}$ & $\begin{array}{c}\text { Não } \\
\text { (Deslocamento } \\
\text { de Elementos } \\
\text { na conversão) }\end{array}$ & & Não \\
\hline
\end{tabular}

Fonte: $\mathrm{O}$ autor

\subsection{Mapeamento APO x BIM}

Nesta etapa do estudo de Campo, realizaou-se o mapeamento das informações resultantes da APO (formulários) e BIM (modelo de informação) onde, para esta etapa inicial da pesquisa, optou-se por utilizar somente o formulário destinado as unidades habitacionais, 
composto por um total de 68 questões (não sendo utilizado o formulário referente às áreas comuns e equipamentos externos).

A partir da classificação destas 68 questões em três categorias de acordo com a natureza das questões e seus objetivos observou-se 3 classificações, a saber: Identificação (identificação dos avaliadores, da unidade habitacional, identificação dos entrevistados,), Satisfação (percepção e satisfação do ambiente construído, conforto, mobilidade, uso e manutenção) e Patologias (manifestações patológicas).

As Zonas são unidades espaciais do modelo, representando visualmente este espaço, (plano bidimensional e tridimensional) sendo possível avaliar propriedades físicas deste espaço (metragens, informações de ocupação e uso, etc.), como também avaliações energéticas (conforto térmico, insolação, iluminação, etc.). Uma capacidade única de reconhecer as portas e janelas que estejam dentro dela é uma característica única da ferramenta zona, desta forma, permitindo não somente a introdução das questões referentes a identificação e satisfação, como também introduzir as questões relacionadas a patologias da edificação.

Outra observação importante é a facilidade oferecida pela ferramenta zona para se criar propriedades IFC diretamente em sua caixa de configuração através do Gestor de Propriedades IFC. Através deste procedimento as 68 questões da APO serão inseridas como se fossem propriedades IFC dos objetos ZONA.

Inicialmente, as 68 questões serão inseridas uma a uma, em apenas um objeto Zona, que depois será copiado e distribuídos para cada uma das unidades do conjunto habitacional (Figura 5).

\section{Figura 5 - Quadra do Campinas F com Zonas em 3D e caixa de setagem do Gestor de propriedades IFC}

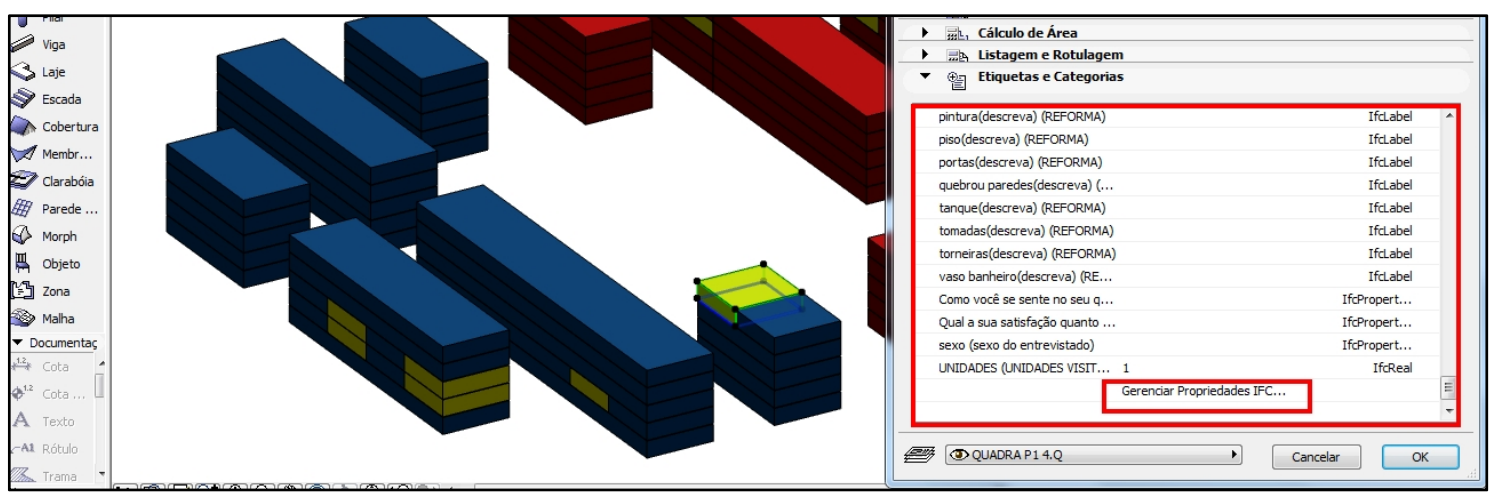

Fonte: $\mathrm{O}$ autor

Com cada uma das unidades abastecidas com o objeto zona com as questões inseridas, o modelo se encontra pronto para ser levado a campo e receber diretamente na lista gerada as respostas dos entrevistados na fase de condução da APO.

A partir do preenchimento dos campos destinados as respostas na lista gerada pelo modelo .PLN, é possível gerar imediatamente um arquivo. XLS (Microsoft Excel), eliminando desta forma, no processo de entrega de dados, o procedimento de compilação manual das informações coletadas a campo (digitalização das respostas em planilha Excel), simplificando o processo e aumentando o grau de confiabilidade e segurança das informações coletadas a campo (entrevistas) (Figura 6). Mantém se a organização da tabulação anterior, onde a linha do cabeçalho refere-se a cada questão do formulário da APO, e cada linha subsequente corresponde as respostas de cada entrevistado, podendo ser alteradas diretamente na interface e exportada automaticamente em .xls. 
Figura 6 - Interface lista de modelo.PLN e planilha .xls gerada

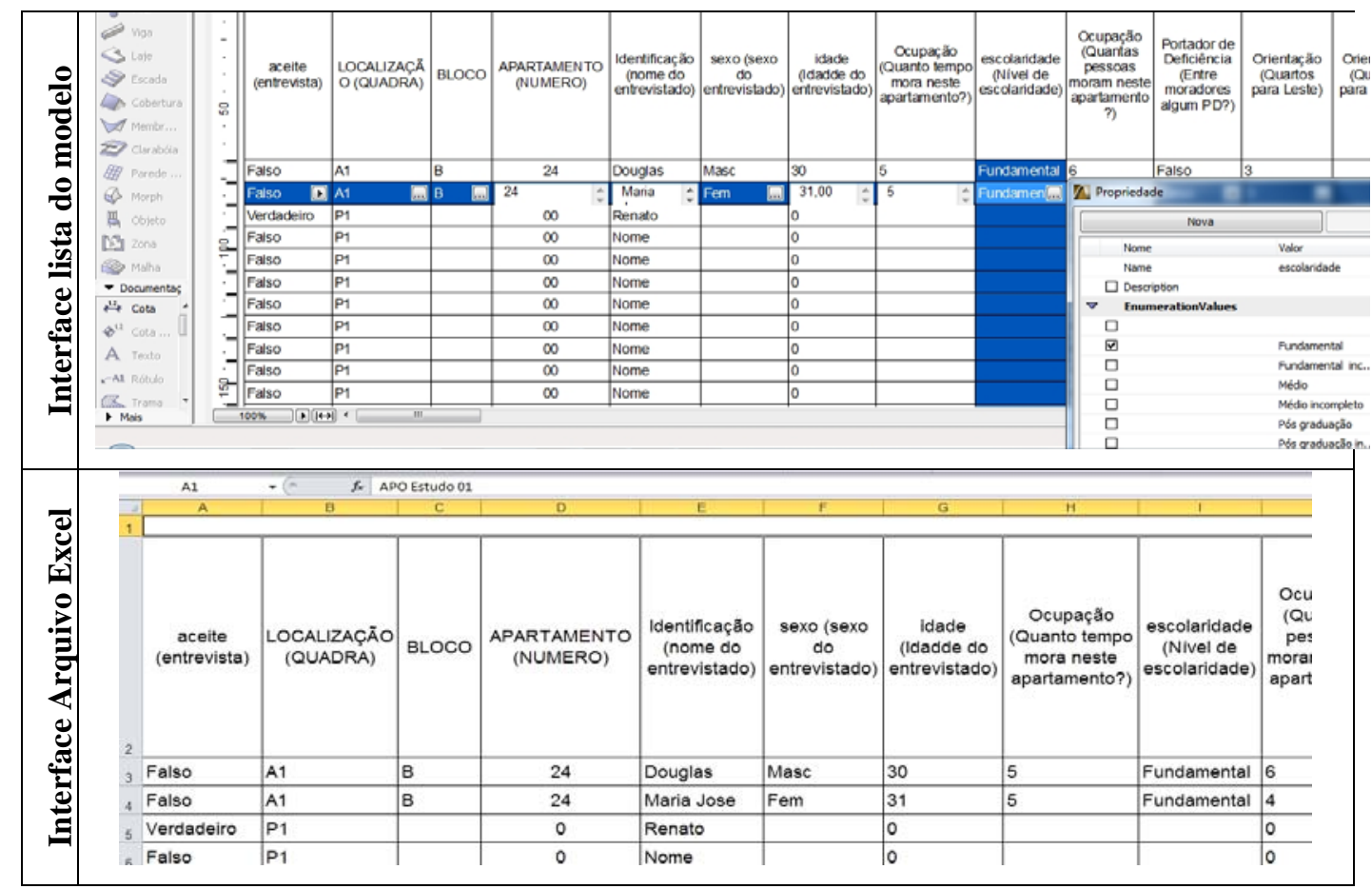

Fonte: $\mathrm{O}$ autor

\subsection{Prova de conceito}

Neste experimento um conjunto de informações objetivas e subjetivas (questões da APO) foi inserido e distribuído como propriedades de objetos Zona, no modelo paramétrico do conjunto habitacional Campinas - F. Este conjunto de informações pode agora ser organizado, manuseado de forma objetiva, gerando listas (planilhas) específicas, de acordo com a conveniência, necessidade de consulta, para contribuir nas tomadas de decisões projetuais em qualquer momento ou fase do processo de projeto. Em um segundo momento foram gerados arquivos. XLS para testar a estabilidade de comunicação e troca de arquivos da plataforma Archicad e Microsoft Excel,. Não é possível a importação de arquivo. xls, mas os arquivos exportados, se apresentam íntegros e consistentes. Também foram gerados e exportados arquivos. IFC que o foram analisados no software Solibri e demonstraram estabilidade, consistência onde as propriedades criadas se mantiveram íntegras.

\section{CONSIDERAÇÕES FINAIS}

Os resultados obtidos até o momento são satisfatórios, respondendo as questões iniciais da pesquisa. Até agora a pesquisa responde satisfatoriamente sobre como introduzir as questões da APO em modelo de informação, o quanto destas questões o modelo é capaz de absorver e quais os produtos nesta fase. Comparando a elaboração de APO convencional com a elaboração de APO suportada por BIM, pode-se observar que o fluxo da informação passa por significativa alteração (Figura 7), oferecendo um fluxo mais enxuto e direto entre a fonte destas informações (usuários) e projetistas (responsável pelas tomadas de decisões projetuais). Também foram avaliados os produtos possíveis de serem gerados e integrados ao ciclo da APO (planilhas Excel, arquivos. IFC), constatando a manutenção de integridade dos formulários introduzidos e convertidos em propriedades do objeto zona. 
Estes resultados, a primeira vista parecem simples, mas avaliando a inexistência de ferramenta BIM específica para APO, podem ser estes procedimentos aqui demonstrados e sugeridos, a base de desenvolvimento de plugins ou ferramentas específicas para essa aproximação do BIM com a APO.

\section{Figura 7 - Fluxo de informação: APO tradicional (esquerda) e APO com BIM (direita)}

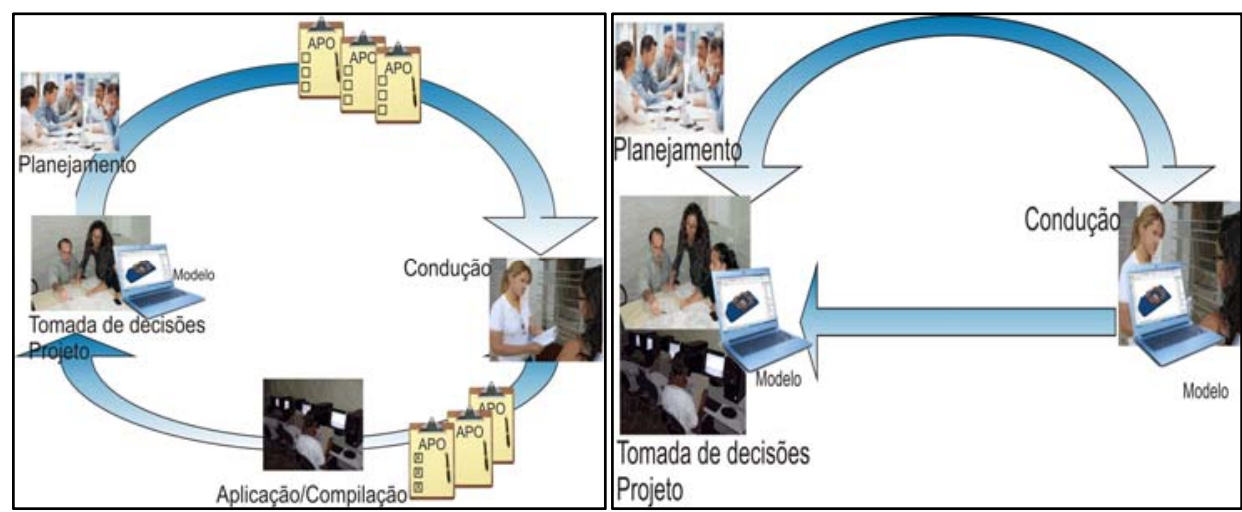

Fonte: $\mathrm{O}$ autor

\section{AGRADECIMENTOS}

Ao Conselho Nacional de Desenvolvimento Científico e Tecnológico (CNPq), processo $n^{0} 381052 / 2012-5$, pela bolsa de Desenvolvimento Tecnológico e Industrial e à Fundação de Amparo à Pesquisa do Estado de São Paulo (FAPESP), processo n ${ }^{0}$ 2014/16362-1, pelo apoio na divulgação da pesquisa.

\section{REFERENCIAS}

FUJIMORI, M. I. B. Resultados da avaliação pós-ocupação no conjunto habitacional Campinas - F. Campinas, 2012. 84f. Monografia (Iniciação Científica) - Faculdade de Engenharia Civil, Arquitetura e Urbanismo, Universidade Estadual de Campinas, Campinas, 2012.

EASTMAN, C. Building Product Models: computer environments supporting design and construction. Boca Raton: CRC Press, 1999.

EASTMAN, C. et al. Bim Handbook: A Guide to Building Information Modeling for Owners, Managers, Designers, Engineers and Contractors. Hoboken (NJ) : John Wiley \& Sons, 2008.

GIL, A. C. Como elaborar projetos de pesquisa. 4 ed. São Paulo: Atlas, 2002.

GRANJA et al. A natureza do valor desejado na habitação social. Revista

AmbienteConstruído. Porto Alegre, v. 9, n. 2, p. 87-103, abr./jun. 2009. Disponível em: http://www.antac.org.br/AmbienteConstruido/pdf/revista/artigos/Doc183262.pdf. Acesso:15/05/ 2010.

KOWALTOWSKI, C.C.D, et. al. Análise de parâmetros de Implantação de conjuntos habitacionais de interesse social: ênfase nos aspectos de sustentabilidade ambiental e qualidadede vida. In FINACIADORA DE ESTUDOS DE PROJETOS. Construção e Meio Ambiente.Brasília: FINEP, 2006. v. 7, Cap. 5, p.128-167. (coletânea Habitare). 
LEAMAN, A.; STEVENSON, F.; BORDASS, B. Building evaluation: practice and principles. Building Research \& Information, London, v. 38, n. 5, p. 564-577, Aug. 2010

ORNSTEIN, S. W.; ROMÉRIO, M. D. A. Avaliação pós-ocupação do ambiente construído. São Paulo: Studio Nobel, 1992.

PREISER, W. F. E.; RABINOWITZ, H. Z.; WHITE, E. T. Post-occupancy evaluation. [S.1.]: Van Nostrand Reinhold, 1988.

SILVA, M. A. C.; SOUZA, R. D. Gestão do processo de Projeto de Edificações. 1. ed. São Paulo: O Nome da Rosa, 2003.

SUCCAR, B. Building Information Modelling Framework: A research and delivery foundation for industry stakeholders. Automation in Construction, 2009.

WEBSTER, A. E. A. Augmented Reality in Architectural Construction, Inspection, and Renovation. CONGRESS ON COMPUTING IN CIVIL ENGINEERING, Anaheim, jun 1996. $1-7$.

ZIMRING, G. Postoccupancy Evaluation: Issues and Implementations. In: BECHTEL, R.; CHURCHMAN, A. Handbook of Environmental Psychology. New York: John Wiley \& Sons, Inc., 2002, 306-319 